\title{
Effect of Noise Factor on System Performance in LTE Networks
}

\author{
Mohana H.K. \\ Seshadripuram First \\ Grade College, \\ Yelahanka, Bangalore \\ India
}

\author{
Mohankumar N.M. \\ Dept. of Electronic \\ Science, Bangalore \\ University, Bangalore, \\ India
}

\author{
Suhas K.R. \\ Dept. of Electronic \\ Science, Bangalore \\ University, Bangalore, \\ India
}

\author{
Devaraju J.T. \\ Dept. of Electronic \\ Science, Bangalore \\ University, Bangalore, \\ India
}

\begin{abstract}
The 3GPP Long Term Evolution (LTE) technology provides higher system throughput over Broadband Wireless Access (BWA) telecommunication systems in order to meet the escalating demands of multimedia services. In such systems, the higher noise factor at the base station (eNB) degrades the system throughput, since the increase in noise factor at the eNB decreases the Signal to Noise Ratio (SNR) of the received signal. Thus the network deployment with lower noise factor at the eNB support higher system throughput and it is essential to provide high Quality of Services (QoS) in LTE networks. Hence in this paper, an attempt has been made to study and evaluate the effect of various noise factors at the eNB on system performance in uplink LTE network using QualNet 7.1 network simulator. The performance metrics considered for the simulation studies are spectral efficiency, system throughput, total numbers of data bytes received, total numbers of transport blocks received with errors, delay and jitter.
\end{abstract}

\section{General Terms}

Noise Factor, LTE, Radio Range, Transport Block, Simulation

\section{Keywords}

3GPP, Spectral Efficiency, Throughput, Data Bytes Received, Delay, Jitter, SNR, MCS

\section{INTRODUCTION}

The growth in the use of Broadband Wireless Access telecommunication systems employs Third Generation Partnership Project (3GPP) LTE technology due to its higher system throughput, increased system capacity, reduced latency, bandwidth flexibility, greater coverage, seamless connectivity, reduced power consumption, flexibility in the network deployment, lower cost of network construction and operation [1]. Thus the escalating demands of multimedia, voice and data services of the BWA telecommunication systems are fulfilled by the higher system throughput. The system throughput of such network reduces when the noise factor at the eNB increases, since the increase in noise factor at the eNB increases the amount of noise added to the signal by the system. Hence the noise factor at the eNB plays a significant role on system performance [2]. Thus, the network deployment with the lower noise factor $(\geq 1)$ at the eNB allows the system to add less noise to the signal and it is essential in network to increase the SNR value of the received signal to achieve higher system throughput for better QoS. Hence in this paper, the performance of uplink LTE system is evaluated for various noise factors at eNB using QualNet 7.1network simulator. The rest of this paper is organized as follows. Section 2 gives a brief insight of related work. Simulation studies and results are given in section 3 and Section 4 gives the future scope of this work and section 5 concludes the paper.

\section{RELATED WORK}

In wireless transmission infrastructure, the quality of a signal received by a particular User Equipments (UEs) for high quality services depends on the noise factor at the eNB, position of UEs from the eNB, multipath fading etc. The lower noise factor at the eNB causes high quality radio link environment in order to support higher data rates to achieve higher system throughput, which is essential for better QoS. The noise factor of such a network defined as the ratio of SNR at the input to the SNR at the output and is related by the equation [3]

$$
\mathbf{F}=\frac{\mathbf{S N R}_{\text {in }}}{\mathbf{S N R}_{\text {out }}}
$$

Thus in network, the noise factors gives the indication of signal power density to noise power density at the input and output and is represented by [3, 4]

$$
\mathrm{F}=\frac{\left(S_{i} / N_{i}\right)}{\left(S_{o} / N_{o}\right)} \geq 1
$$

In BWA telecommunication systems the noise factor value becomes 1 when the noise added by the system is zero. Thus, the noise factor is a number which specifies the performance of the system. The minimum possible noise factor at the eNB $(\geq 1)$ provides maximum system throughput for better QoS [3, 4]. Also in system the increase in noise factor at the eNB increases the noise added to the received signal and is related by the equation $[2,5]$

$$
\mathbf{N}_{\text {Total }}=\mathbf{F} \times \mathbf{N}_{\mathbf{o}}
$$

Where, $\mathrm{N}_{\mathrm{o}}$ is the background noise or random noise generated by all matters. Further, the receiver sensitivity of the cellular system is the sum of the ambient noise, noise factor at eNB (measures of SNR) and other components in the receiver chain such as feeder cables, jumper cables, lightning protection and duplexer and is given by [6]

$$
\text { Sensitivity }=\mathbf{N}_{0}+\mathbf{N}_{\text {Receiver }}+\mathrm{SNR}
$$

Therefore, in cellular system the increase in noise factor at eNB causes the reduction of system throughput or coverage due to receiving sensitivity degradation [6]. Further, in LTE networks based on the received SNR value the eNB select the higher order Modulation and Coding Schemes (MCS) for data transmission. Thus in LTE network the lower noise factor at eNB allows the higher order MCS to carry data bits due to its higher SNR values and it is essential to increases the system performance by carrying more data bits per hertz [7, 8]. However the amount of SNR value received by the UEs for services depends on its locations. The UEs placed nearby eNB 
are received the signal with higher SNR value due to less path loss. Conversely, as the distance between the UEs and eNB increases the amount of SNR value received by the UEs decreases due to increase in path loss and it significantly decreases the system performance. Thus, in LTE network the noise factor at the eNB and the location of UE in the network playing a significant role on system performance and hence the integrated study of noise factor at the eNB and the location of UEs in the network become vital to analyze system performance.

\section{SIMULATION STUDIES AND RESULTS}

In LTE uplink, the effect of noise factor at the eNB on system performance is evaluated using QualNet 7.1 network simulator by considering an eNB and a single UE in a terrain area of $500 \mathrm{~m} \times 500 \mathrm{~m}$. In this scenario, an uplink CBR connection of data rate $10 \mathrm{Mbps}$ is established between an eNB and a UE with two ray path loss model and Rayleigh fading environment. The remaining simulation parameters considered for the simulation studies are listed in Table 1.

Table 1. Simulation Parameters

\begin{tabular}{|l|l|l|}
\hline \multicolumn{1}{|c|}{ Property } & \multicolumn{1}{c|}{ Value } \\
\hline Simulation-Time & $100 \mathrm{~S}$ \\
\hline Propagation-Channel-Frequency[0] & $2.4 \mathrm{GHz}$ \\
\hline Propagation-Channel-Frequency[1] & $2.5 \mathrm{GHz}$ \\
\hline Propagation-Model & Statistical \\
\hline Shadowing Means & $4 \mathrm{~dB}$ \\
\hline Channel Bandwidth & $10 \mathrm{MHz}$ \\
\hline Antenna Model & Omni directional \\
\hline Antenna Gain(dB) & 0 \\
\hline \multirow{5}{*}{ eNB } & MAC-Scheduler-Type & Proportional Fair (PF) \\
\cline { 2 - 3 } & PHY-Tx-Power (dB) & 15 \\
\cline { 2 - 3 } & Antenna Height & 15 meters \\
\cline { 2 - 3 } & MAC Transmission Mode & Single antenna scheme \\
\cline { 2 - 3 } & PHY-Num-Rx- Aneannas & 2 \\
\hline \multirow{5}{*}{ UE } & MAC-Scheduler-Type & Simple-Scheduler \\
\cline { 2 - 3 } & PHY-Tx-Power (dB) & 10 \\
\hline & Antenna Height & 1.5 meters \\
\cline { 2 - 3 } & PHY-Num-Tx-Aneanna & 1 \\
\hline
\end{tabular}

The snapshot of the scenario designed for the simulation studies using QualNet 7.1 network simulator is shown in Figure 1. Initially, the simulation study is carried out by placing the UE at a distance of $50 \mathrm{~m}$ from the eNB with noise factor of 1 . The performance metrics such as spectral efficiency, system throughput, total data bytes received, total transport blocks received with errors, average delay and average jitter are recorded. Simulation studies are repeated by changing the noise factor at the eNB from 2 to 10 in steps of 1 and 10 to 50 in steps of 10 . The simulation studies are also repeated by placing the UE at a distance of $100 \mathrm{~m}, 200 \mathrm{~m}$, $300 \mathrm{~m}$, and $400 \mathrm{~m}$ from the eNB.

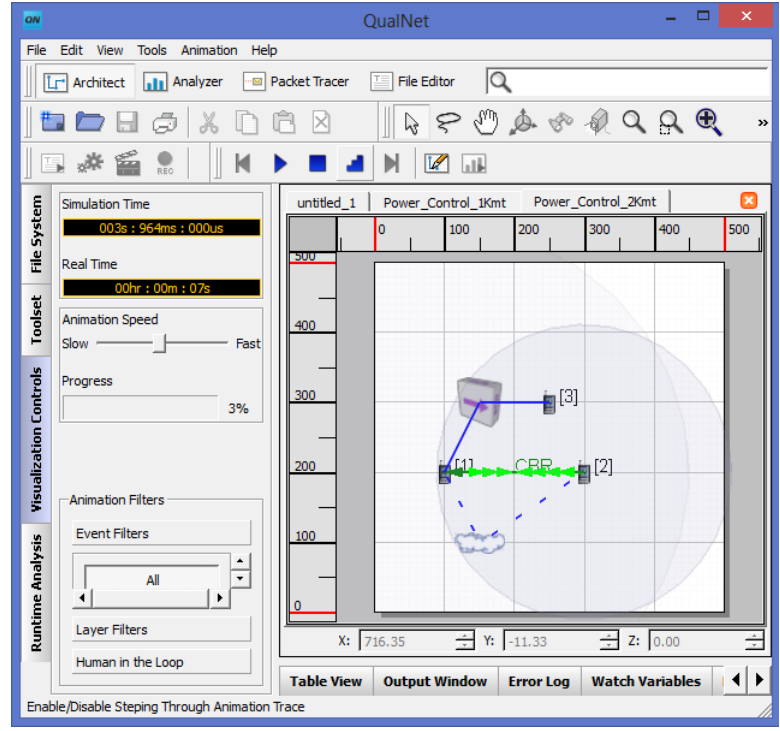

Fig 1: Snapshot of the Scenario designed for simulation study

Figure 2, 3, 4 and 5 shows the spectral efficiency, system throughput, total numbers of data bytes received and total transport blocks received with errors for varying noise factor at the eNB for various UE locations. It is depicted from figure 2, 3 and 4 that the spectral efficiency, system throughput and data bytes received performance is better for UE placed near to the eNB with lower noise factor. Since for nearby UE the path loss is less and for lower noise factor at the eNB the amount of noise added to the received signal is also less and is evident from figure 5 that the total numbers of transport blocks received with errors are less for UE placed nearby eNB with lower noise factor. This causes high quality radio link environment in the network and hence the system selects the higher order MCS to carry more data bits per hertz to achieve higher data rates in order to support higher spectral efficiency and higher system throughput for better QoS $[9,10,11]$. It is also observed from figure 2, 3 and 4 that the increase in distance between the UE and eNB with increase in noise factor decreases spectral efficiency, system throughput and total data bytes received in the network. Because the increase in distance between UE and eNB with increase in noise factor, increases the total numbers of transport blocks received with errors due to reduction in MCS and it causes the degradation of data rates in the network $[7,8]$.

Figure 6 and 7 shows the average delay and average jitter performance for varying noise factor at the eNB for various UE locations. It is evident from figure 6 and 7 that the average delay and average jitter performance are less for UE placed near to the eNB with lower noise factor. Because the lower noise factor at the eNB adds less noise to the received signal, which provides higher SNR value for services to support higher data rates and it radically reduces the queuing delay and jitter. From figure 6 and 7 it is also illustrates that the increase in distance between the UE and eNB with increase in noise factor increases the average delay and average jitter due to lower data rates in the network $[10,12]$. 


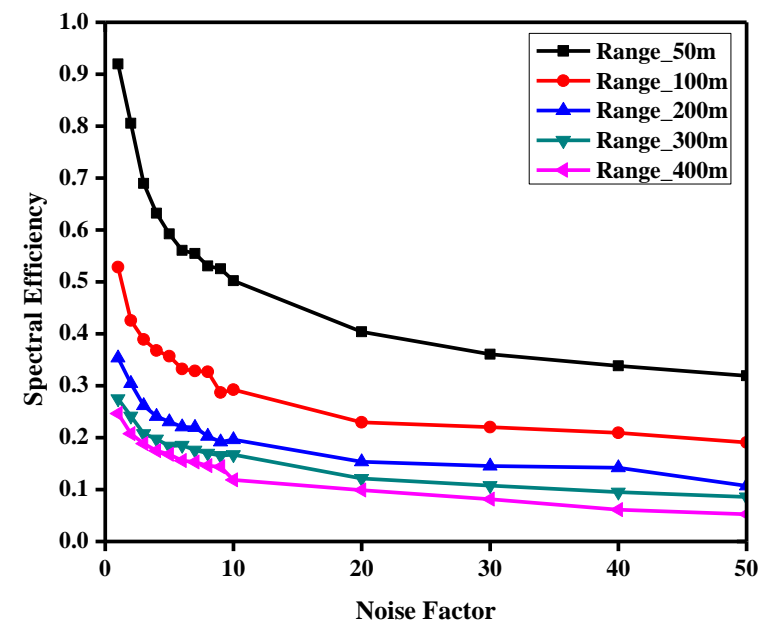

Fig 2: Spectral Efficiency performance for varying noise factor at the eNB for various UE locations

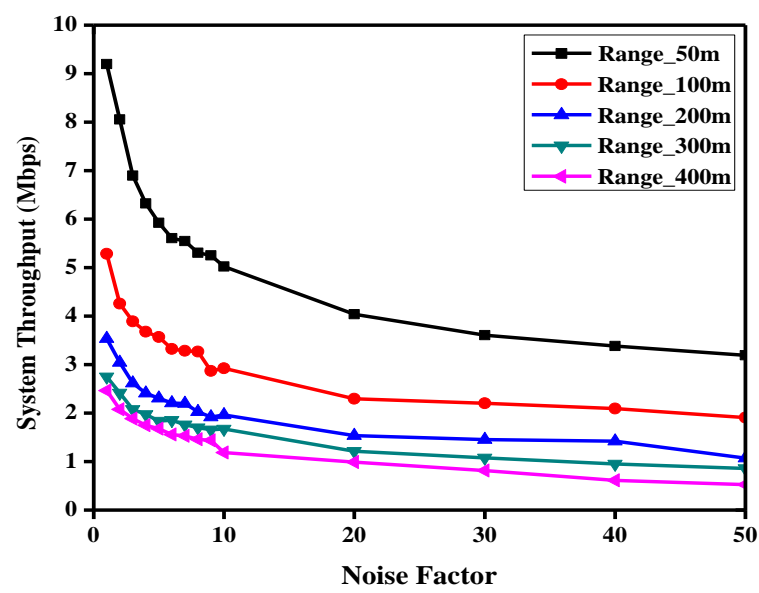

Fig 3: System Throughput performance for varying noise factor at the eNB for various UE locations

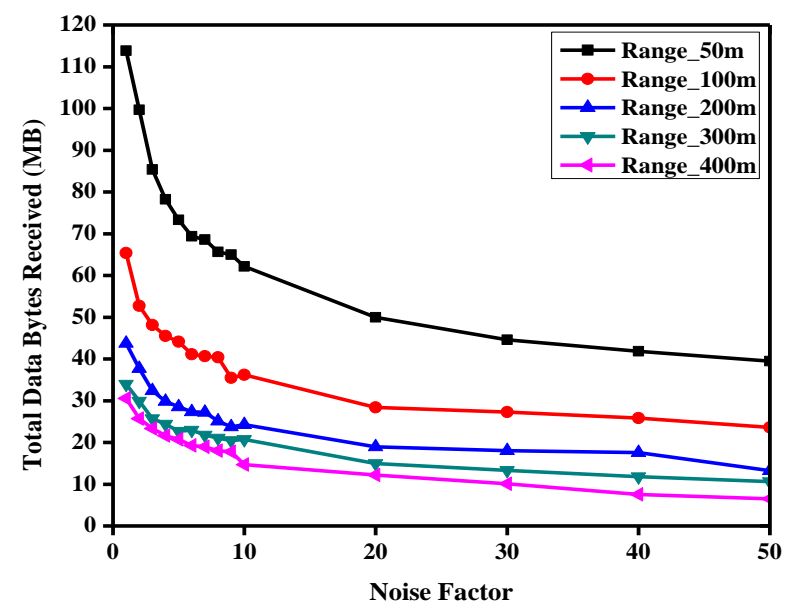

Fig 4: Data Bytes Received performance for varying noise factor at the eNB for various UE locations

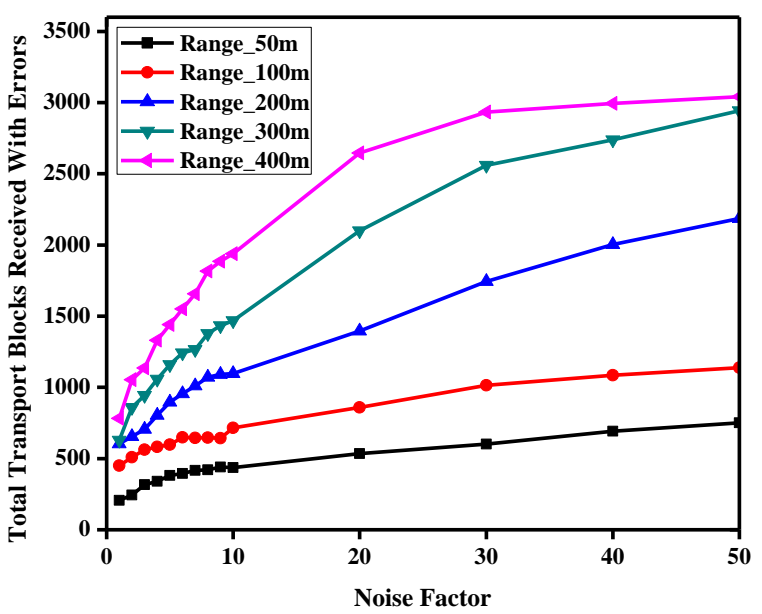

Fig 5: Total Blocks Received with Errors for varying noise factor at the eNB for various UE locations

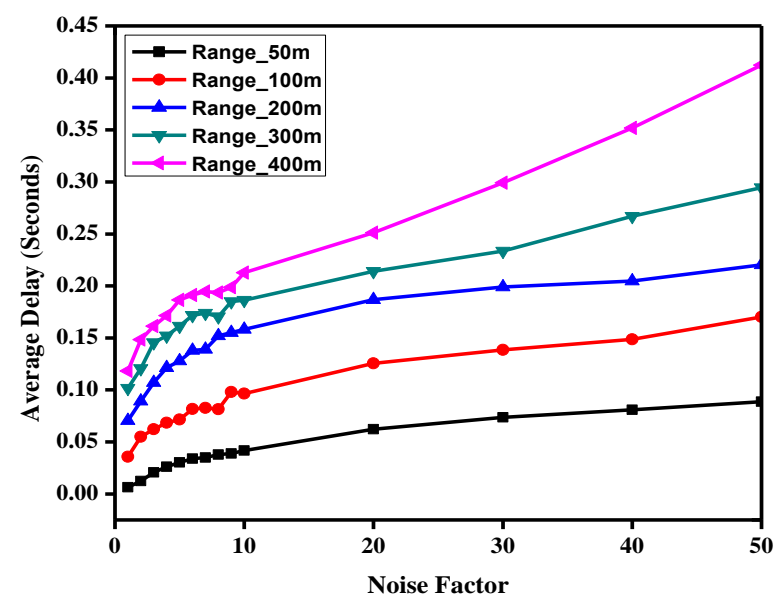

Fig 6: Average Delay performance for varying noise factor at the eNB for various UE locations

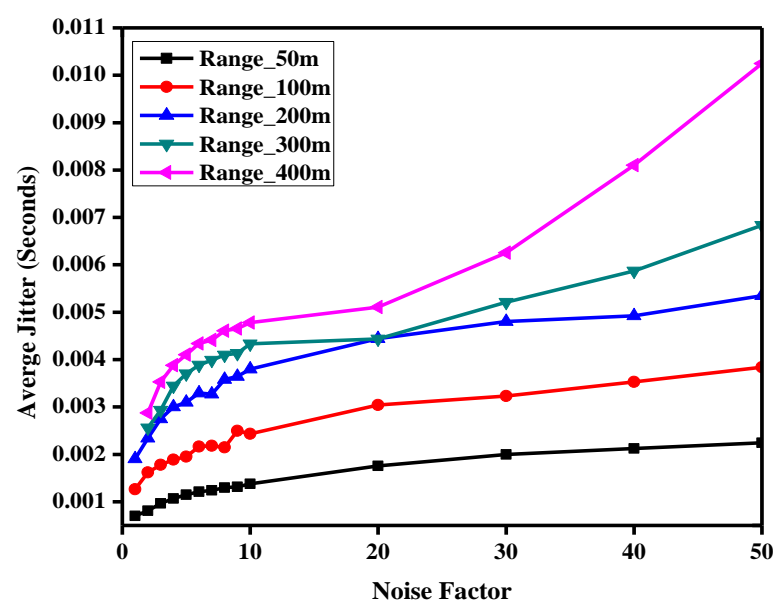

Fig 7: Average Jitter performance for varying noise factor at the eNB for various UE locations 


\section{FUTURE SCOPE}

In network, the lower noise factor at the eNB offers higher SNR value to the received signal to support higher data rate radio signal for better QoS. Thus in 3GPP LTE systems the deployment of network with lower noise factor at the eNB provides higher system throughput and lower latency radio signals for high speed internet, better quality voice and multimedia services.

\section{CONCLUSION}

In this paper, the effect of noise factor at the eNB on LTE system performance is evaluated by using QualNet 7.1 network simulator. The system performance metrics considered for the simulation studies are spectral efficiency, system throughput, total data bytes received, total transport blocks received with errors, average delay and average jitter. The simulation result shows that the system performance is better for UE placed near to the eNB with lower noise factor. Since for nearby UE the path loss is less and for lower noise factor at the eNB the amount of noise added to the received signal is also less, which provides higher SNR radio signal for services. Due to this the eNB selects the higher order MCS to carry data bits in the network and it consequently increases the data rates of the system. Thus in 3GPP LTE system, the network deployment with lower noise factor at the eNB supports high quality voice and multimedia services.

\section{ACKNOWLEDGMENTS}

One of the Authors of this paper would like to thank Director of Studies and Management of Seshadripuram Educational Trust (SET), Bangalore for their support. Authors would like to thank UGC for providing Junior Research Fellowship under 'At Any One Given Time Basis Scheme' to carry out the research work.

\section{REFERENCES}

[1] Jaafar A. Aldhaibani, A.Yahya, R.B.Ahmab, A.S. Md Zain, M.K.Salman, Riad Edan "On Coverage Analysis for LTE-A Cellular Networks", International Journal of
Engineering and Technology (IJET), Vol 5(1) Feb-Mar 2013

[2] Mike Eddy, "Optimizing LTE Network Performance with Tower Mounted Amplifiers", Cell Site Optimization Westell Technologies

[3] Purnachandar Poshala, Rushil KK, Robin Gupta, " Signal Chain Noise Figure Analysis" Texas Instruments Application Report, October 2014

[4] AgilentTechnologies "Agilent Fundamentals of RF and Microwave Noise Figure Measurements", Application Note 57-1

[5] Prof. Murat Torlak, "Path Loss" EE4367 Telecom. Switching \& Transmission

[6] Han-Nien Lin, Feng-Chia University, Taiwan, R.O.C., "Analysis of Platform Noise Effect on Performance ofWireless Communication Devices"

[7] Krishna Balachandran, Member, IEEE, Srinivas R. Kadaba, Member, IEEE, and Sanjiv Nanda, Member, IEEE, "Channel Quality Estimation and Rate Adaptation for Cellular Mobile Radio", IEEE Journal on Selected Areas in Communications, Vol 17(7), July 1999

[8] Wen-Bin Yang, Michael Souryal "LTE Physical Layer Performance Analysis", NISTIR 7986

[9] Ronell B. Sicat, Professor Tareq Y. Al-Naffouri, "Bit Error Probability Computations for M-ary Quadrature Amplitude Modulation"

[10] Mohana H K, Mohankumar N M, Devaraju J T "Impact of Adaptive Modulation and Coding Schemes on Bit Error Rate for System Performance in the Uplink LTE System", International Journal of Computer Technology \& Applications (IJCTA), Vol 6 (5), Sept-Oct 2015

[11] ETSI TS 136213 V10.1.0 (2011-04), "LTE; Evolved Universal Terrestrial Radio Access (E-UTRA); Physical layer procedures (3GPP TS 36.213 version 10.1.0 Release 10)", Technical Specification

[12] Ronak D. Trivedi , M. C. Patel, "Comparison of Different Scheduling Algorithm for LTE", International Journal of Emerging Technology and Advanced Engineering, Vol 4( 5), 2014 